it to be spherical as a first approximation), $A$ is the distance from the galaxy to the nebula, $M$ is mass of our galaxy, and $G$ is the gravitational constant. However, according to the principle of relativity, no mass can acquire a velocity greater than $c$, the velocity of light in vacuo. Consequently, in order to preserve the law of gravitation, together with the conservation of energy involved, and to agree with the principle of relativity the following equation must be satisfied :

$$
h \nu-\mu k\left(1-\frac{R}{A}\right)=\mu c^{2} ; \quad . \quad .
$$

or, substituting $\mu$ by its value from (1), we have

$$
h \nu\left[1-\frac{k}{c^{2}}\left(\mathrm{I}-\frac{R}{A}\right)\right]=\mu c^{2} . \quad .
$$

On the right-hand side of this equation $c$ is constant and, therefore, $\mu$ cannot vary, which makes $\mu c^{2}$ constant. On the left-hand side of the equation, $h$ is Planck's constant. Therefore, in order to satisfy equation (2), $v$ which we observe in the photon coming from the nebula, and which is on the lefthand side of equation (2), must have a value different from $\nu$ which the photon possessed at the moment of emission, and become a variable frequency $\nu^{1}$, a function of the argument $A$.

Equating (2) with (5), we have

$$
h \nu^{1}=h v\left[1-\frac{k}{c^{2}}\left(1-\frac{R}{A}\right)\right] . .
$$

This equation shows that under an acceleration by the field of gravitation a photon keeps its velocity constant by adjusting its frequency so as to compensate for the variation due to the acceleration.

The red shift of the 'expanding universe' is an immediate corollary of equation (6), as the observed wave-length $\lambda^{1}$ is $1 / \nu^{1}$, and, therefore, increases with the distance $A$.

It seems that the idea presented is worth discussion; I should be much interested in any comments on the subject.

Extension Division,

Anatol James ShNerderov.

George Washington University,

Washington, 6, D.C. Nov. 14.

1 Abbott, C. G., "The Newtonian Lucretius", Smithsonian Misc Collections, 142 (1899). Shneiderov, A. J', "The Exponentia Law of Gravitation and Its Effects on Seismological and Tectonic Law of Gravitation and Its Effects on Seismological

\section{Surface Tension of Solutions}

In a recent paper ${ }^{1}$, Wales discusses the approximate relations for the surface tension of regular solutions. $\mathrm{He}$ concludes that the surface tension is linearly or quadratically related for ideal and regular solutions respectively to the molar volume fractions of the components.

Two facts, however, limit the applie bility of these relations. (1) The omission to take into account the adsorption at the surface which is responsible for the disagreement between the relations obtained and the Gibbs equation for the surface tension. (2) The neglect of the orientation of molecules at the surface.
In a theory I have recently developed ${ }^{2}$ both those effects are accounted for in terms of the monolayer, while the derived relations for the surface tension agree perfectly with the Gibbs equation and cover the whole range of concentrations. At close values of the surface tension, relations analogous to those derived by Wells are obtained, while the surface tension for ideal solutions is linearly related to the surface molar fraction, which indicates the area occupied by molecules of the given species in the monolayer. An inspection of the benzene-carbon disulphide system, discussed by Wells, shows the carbon disulphide molecule to be oriented perpendicular to the surface.

A paper devoted to the statistical treatment of regular solutions and, in particular, to the analysis of the benzene-carbon disulphide system was ready in February 1944 and is to appear shortly in Acta Physicochimica USSR.

\section{A. A. SchuChow ITzky.}

'J. Chem. Phys., 12, 134 (1944).

'Acta Physicochim. USSR, 19, 176 (1944).

\section{Plant-Growth Substances and Penicillium notatum}

IT was considered of interest to study the effect of plant-growth substances on the production of penicillin by the mould $P$. notatum. Several experiments have been carried out using indole-3-acetic acid and $\alpha$-naphthalene acetic acid. The study has included the use of various media with the Squibb strain of $P$. notatum (American Type Culture Collection). Heavy spore inocula were added to two quart bottles (flat type) containing about $300 \mathrm{ml}$. of the medium. The bottles after inoculation were kept at $23-24^{\circ} \mathrm{C}$. and 80 per cent humidity. As might be expected, marked stimulatory effects of the growth-substances were found only in the cases of the simple media, such as Czapek-Dox with added brown sugar. The experiments reported refer to penicillin production in the latter medium with 4 per cent brown sugar. Addition of either of these growthsubstances at the concentration of 1 part in 30,000 had no effect in a mineral and corn-steep ( 6 per cent) medium. The titres obtained with the simple medium plus the growth substances are higher than have previously been reported for this medium alone. The practical value of the findings is limited, however, for much higher titres are the rule with the corm-steep media. This work suggests that a part of the good results got with the corn-steep may be ascribed to its containing plant-growth stimulants; but this point has not been checked.

The results of repeated tests have been somewhat erratic because of the variation in bottles inoculated under identical conditions. Almost without exception, however, the bottles containing the growth substances have developed mature mould mats in somewhat shorter times, and the titres of penicillin (both at the same time and at comparable stages during the development of the mat) have been higher than in controls. It is not known whether this is due to a faster growth or to a greater total growth of the mould, or to an increased secretion of penicillin. It is thought that faster growth of the mat will account for the findings, for, of course, the quicker the peak concentration of penicillin is reached, the higher it will be, there being less time for the decomposition of the accumulating penicillin. 